\title{
Mechanisms for Discordant Alternans
}

\author{
MARI A. WATANABE, M.D., Ph.D., FlAVIO H. FENTON, Рн.D.,* \\ STEVEN J. EVANS, M.D., $\dagger$ HAROLD M. HASTINGS, Ph.D.,* ALAIN KARMA, Рн.D.
}

From the Physics Department, Northeastern University, Boston, Massachusetts; the *Mathematics Department, Hofstra University, Hempstead, New York; and the †Cardiology Department, Beth Israel Hospital, New York, New York

\begin{abstract}
Discordant Alternans Mechanism. Introduction: Discordant alternans has the potential to produce larger alternans of the ECG $T$ wave than concordant alternans, but its mechanism is unknown.

Methods and Results: We demonstrate by one- and two-dimensional simulation of action potential propagation models that discordant alternans can form spontaneously in spatially homogeneous tissue through one of two mechanisms, due to the interaction of conduction velocity and action potential duration restitution at high pacing frequencies or through the dispersion of diastolic interval produced by ectopic foci. In discordant alternans due to the first mechanism, the boundaries marking regions of alternans with opposite phase arise far from the stimulus site, move toward the stimulus site, and stabilize. Dynamic splitting of action potential duration restitution curves due to electrotonic coupling plays a crucial role in this stability. Larger tissues and faster pacing rates are conducive to multiple boundaries, and inhomogeneities of tissue properties facilitate or inhibit formation of boundaries.

Conclusion: Spatial inhomogeneities of electrical restitution properties are not required to produce discordant alternans. (J Cardiovasc Electrophysiol, Vol. 12, pp. 196-206, February 2001)
\end{abstract}

discordant alternans, $T$ wave alternans, dynamic restitution, restitution

\section{Introduction}

Occurrences of ECG T wave alternans (TWA) were noted soon after the first ECGs were published 90 years ago, but such overt TWA was rare enough to elicit case reports. ${ }^{1}$ Overt TWA frequently was associated with profibrillatory conditions such as angina, acute myocardial ischemia (reviewed by Verrier and Nearing ${ }^{2}$ ), and long QT syndrome. ${ }^{3}$ Recent advances in signal processing techniques led to the detection of microscopic (microvolt level) TWA from the body surface ${ }^{4}$ and to the discovery that such microscopic TWA is a fairly common phenomenon at high heart rates. Furthermore, clinical studies suggest that there is a high correlation between large amplitude microscopic TWA and sudden cardiac arrest. ${ }^{5,6}$

Despite growing empiric evidence that microscopic

This study was supported by American Heart Association Grant in Aid 96009660 and NIH SCOR in Sudden Cardiac Death 1PL50HL-52319. Dr. Watanabe was supported by NIH National Research Service Award F32 HL10308-01. Dr. Fenton was supported by the Chasanoff Endowment of LIJ Medical Center, Guidant, Medtronic, the Mike and Louise Stein Philanthropic Fund, and the Rosalyn S. Yalow Foundation for Medical Research.

Address for correspondence: Alain Karma, Ph.D., Physics Department, Dana Building, Northeastern University, Boston, MA 02115. Fax: 617-373-2943; E-mail: karma@ presto.physics.neu.edu

Manuscript received 25 May 2000; Accepted for publication 12 October 2000 .
TWA is an important clinical measure of cardiac electrical instability, its exact mechanism is unproven. Alternans of action potential duration (APD) and refractory period is well known to occur in cardiac tissue at high rates, so TWA could well be an ECG manifestation of APD alternans. Alternatively, conduction block occurring every other beat resulting in alternating conduction patterns and alternating size of excited tissue also could produce TWA. ${ }^{7}$ A recent optical mapping study by Pastore et al. ${ }^{8}$ elegantly demonstrated a relationship between discordant alternans associated with TWA and arrhythmia induction. Discordant alternans is the phenomenon of two spatially distinct regions exhibiting APD alternans of opposite phases. As such, it has the potential to produce larger alternans of the ECG $\mathrm{T}$ wave than concordant alternans. In the study by Pastore et al., ${ }^{8}$ several mechanisms for discordant alternans were speculated upon. The purpose of the current study is to present a mechanism for discordant alternans initiation and stabilization using ion channel models and nonlinear dynamics theory. We demonstrate two scenarios. The first scenario involves an ectopic premature stimulus that initiates discordant alternans immediately. In the second scenario, discordant alternans develops slowly after a sinus rate increase at a single site and involves complex interaction of APD and conduction velocity. Most of the analysis is devoted to the second scenario. A significant feature of the mechanism we propose is that it does not require spatial inhomogeneities of cardiac tissue properties. 


\section{Methods}

The Beeler-Reuter (BR) ion channel model ${ }^{9}$ was applied to cables (one dimension) and sheets (two dimensions) of cardiac tissue. All of the tissue adhered to the same ionic equations, except when we varied a calcium current parameter over space to study the effects of tissue inhomogeneity. We used the forward Euler method to solve the partial differential equations in the model, with step-sizes of $0.25 \mathrm{~mm}$ and $0.02 \mathrm{msec}$ in the cables, and $0.125 \mathrm{~mm}$ and $0.03 \mathrm{msec}$ in the sheets. APD restitution refers to the dependence of APD on preceding diastolic interval (DI). Conduction velocity restitution refers to the dependence of conduction velocity on preceding DI. Both APD and conduction velocity restitution have been shown to be monotonically increasing curves in the BR model as measured by S1S2 stimulation protocols. These curves can be found in Fenton and Karma. ${ }^{10}$ We also obtained dynamic restitution curves in our simulations by plotting APD against preceding DI at all sites for a given beat. APD and DI were measured as time spent by a particular location above and below the membrane potential of $-60 \mathrm{mV}$, respectively. Conduction velocity was calculated from the time taken by a depolarization wavefront to travel a distance of $1 \mathrm{~mm}$. The mechanism of discordant alternans initiation and evolution that we propose is simple, so we expected that our results would be applicable to any mathematical or ion channel model that manifested APD and conduction velocity restitution of sufficient slope. To confirm this assumption, in addition to the BR model, we simulated discordant alternans in the Noble ion channel model ${ }^{11}$ and in two- and threevariable mesoscopic models in which restitution parameters could be varied over various physiologic regimes. ${ }^{10,12}$ However, to maintain consistency, all figures show results of BR model simulations with the exception of Figure 2.

\section{Terminology}

Discordance: If alternans in all areas of the tissue under observation are in phase with the alternans at the stimulus site, alternans are said to be concordant. If there are areas of the tissue alternating out of phase with alternans at the stimulus site, the alternans are said to be discordant. Basic cycle length (BCL): Time between pacing stimuli. Cycle length $(C L)$ : Time between two action potential upstrokes. Cycle length is not synonymous with basic cycle length, because time from stimulus to action potential upstroke depends on distance and conduction velocity between the pacing site and the recording site. APD node: A location at which two consecutive beats have the same APD. DI node, conduction velocity node, and CL node were defined similarly.

\section{Results}

\section{Basic Mechanism of Discordant Alternans Initiation}

Discordant alternans is a subset of alternans. In theory, sustained alternans can be produced by pacing tissue with an APD restitution curve that has a maximum slope $>1$, above a threshold rate (see Discussion). Restitution curves in all of the models we studied have regions where the slope is $>1$ and, as expected, produce APD alternans at high rates. The question is, how can discordant alternans arise from concordant alternans? The trivial answer, well understood by alternans researchers, is to have spatial variation of DI that spans DI*, where DI* is the value of DI that satisfies (DI) + (APD following that DI) $=$ pacing cycle length. Any time there is some area of cardiac tissue that has DI $<$ DI*, DI on the next beat will be $>\mathrm{DI}^{*}$, and any time there is some area of cardiac tissue where DI $>$ DI*, DI on the next beat for that area will be $<\mathrm{DI}^{*}$. Therefore, if cardiac tissue simultaneously has areas with DI both $<\mathrm{DI}^{*}$ or $>\mathrm{DI}^{*}$, then on the next beat, the relative sizes of the DI must invert, producing discordant alternans. In other words, initiation of discordant alternans is merely a matter of producing $\mathrm{DI}<\mathrm{DI} *$ or $>\mathrm{DI}^{*}$ at a given moment in time at different regions in space.

Figure 1 illustrates two possible scenarios for producing such an initial DI spatial distribution and consequently discordant alternans. The first, which we call the ectopic focus scenario, is illustrated in the upper panel. The vertical axis corresponds to the cardiac cable (space), and the horizontal axis denotes time, in an orientation similar to a ladder diagram. Action potential traces from 75 positions on an $8-\mathrm{cm}$ cable are shown. The cable was paced at the top end at $\mathrm{BCL}=310 \mathrm{msec}$ to simulate excitations from the sinus node after one ectopic stimulus was given at the bottom end. The sinus beat before the ectopic beat is shown at the left edge of the figure. The ectopic stimulus was delivered with short coupling interval, but not so short as to affect conduction velocity significantly. The ectopic excitation traveled up the cable and collided with the sinus excitation. Although conduction velocity was essentially constant and the tissue was homogeneous, the increasing DI in the upward direction produced increasing APD in the upward direction. This gradient, in turn, produced increasing DI and APD in the downward direction when the next sinus excitation traveled down the cable. Study of the action potential traces shows that the sinus excitations produced a short-long APD alternans at the top, and long-short APD alternans at the bottom of the cable. In other words, a single ectopic excitation was able to generate discordant alternans immediately. Characteristics of the discordant alternans, however, depended in a complex manner on the pacing rate and timing of the ectopic stimulus. If "sinus" rate was slow, the sinus end of the cable showed no alternans, the ectopic stimulus end of the cable showed APD that was alternately larger or smaller than APD at the sinus end, and this semidiscordant alternans was transient. At intermediate rates at which the cable would have displayed only concordant alternans in the absence of an ectopic excitation (see sinus node scenario following), discordant alternans arose if the ectopic excitation collided with the sinus excitation with longer APD. At fast pacing rates, it was found that discordant 


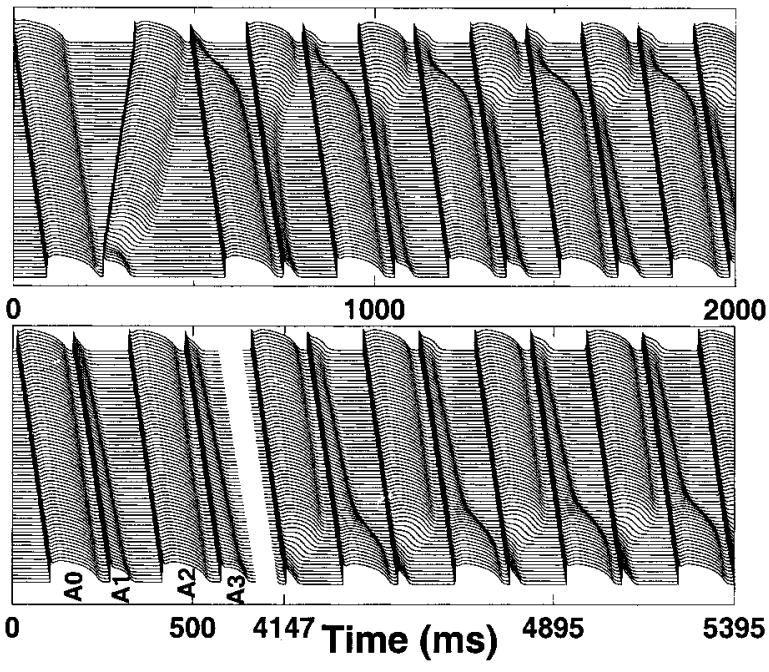

Figure 1. Top: Ectopic focus scenario of discordant alternans initiation. The vertical axis denotes length of a cardiac cable $(8 \mathrm{~cm})$ and the horizontal axis denotes time. Action potential traces from 75 positions are shown. The cable was in quiescence until the first stimulation at the top end of the cable. A stimulus was given $310 \mathrm{~ms}$ after the arrival of the first excitation at the bottom end of the cable to simulate an ectopic focus firing. The ectopic excitation traveled up the cable and collided with the excitation from the second stimulus given at the top. Stimuli were thereafter given at 310-ms intervals at the top end of the cable to simulate sinus excitations. Although conduction velocity was essentially constant and the tissue was homogeneous, the increasing diastolic interval (DI) in the upward direction produced increasing action potential duration (APD) in the upward direction. This gradient, in turn, produced increasing DI and APD in the downward direction when the next sinus excitation traveled down the cable. This inversion continued and alternans was discordant. Bottom: Sinus node scenario of discordant alternans initiation. Orientation as in top panel. All stimuli were applied at the top end of the cable at 310-ms intervals following a long quiescence. The second stimulus followed the maximum possible APD, and DI was short. The short DI produced slow conduction that gave rise to increasing DI as the wavefront traveled down the cable, as seen in the mild increase in the DI preceding the excitation marked $A 1$ and in the mild increase in APD (A1). The third stimulus followed a relatively long DI, producing maximum conduction velocity down the initial segments of the cable. Fast conduction coupled with APD increase down the cable on the previous beat produced decreasing DI and APD (A2) down the length of the cable. The reversal of DI spatial distribution (increasing down the cable for $\mathrm{S1}_{2}$, decreasing for $\mathrm{Sl}_{3}$ ) was exaggerated as pacing continued, and gradually formed a node. The right part of the figure begins with the 13th excitation, and the top and bottom of the cable are clearly alternating out of phase.

alternans arose spontaneously even in the absence of ectopic excitation.

The lower panel in Figure 1 illustrates this second scenario, which we call the sinus node scenario. Stimuli were delivered at the top end of the cable at $310-\mathrm{msec}$ intervals following a long quiescence. BCL was chosen such that the second stimulus followed a short DI. Short DI produced slow conduction as expected from conduction velocity restitution. Slow conduction gave rise to increasing DI as the wavefront traveled down the cable, as seen in the mild increase in DI preceding the excitation marked A1, and in the mild increase in APD (A1). The third stimulus followed a relatively long DI, producing maximum conduction velocity down the initial segments of the cable. Fast conduction coupled with APD increase down the cable on the previous beat produced decreasing DI and APD (A2) down the length of the cable. The difference between maximum and minimum DI over the cable became more pronounced as pacing continued, until a DI node (location where DI is the same on two consecutive beats) appeared. In the figure, the DI node is about one fifth of the way from the bottom trace for the two excitations beginning at time $4,147 \mathrm{msec}$. All points between the stimulus site and the node alternate in phase with the stimulus site, and points beyond the node are out of phase. The node therefore marks the boundary of different phases of alternans and existence of discordant alternans. The DI node gradually moved toward the stimulus site with continued pacing.

In Figure 1, we showed that DI, APD, and conduction velocity were interrelated. In Figure 2, we show how changes in conduction velocity restitution affect the second and third DI spatial distributions. They were computed numerically using the relation $\mathrm{DI}=\mathrm{CL}-\mathrm{APD}$, and that $\mathrm{CL}$ at a given site is $\mathrm{BCL}$ at the stimulus site incremented or decremented by times to local activation, i.e., with notations $\mathrm{A} 0, \mathrm{~A} 1$, and $\mathrm{A} 2$ representing $\mathrm{APD}$ indexed as in Figure 1, and D0, D1, and D2 representing DI preceding those APD:

$$
\begin{aligned}
\mathrm{D} 1(\mathrm{x}) & =\mathrm{CL}(\mathrm{x})-\mathrm{A} 0 \\
& =\mathrm{BCL}+\int_{0}^{\mathrm{x}}\left[\frac{1}{\theta\left(\mathrm{D} 1\left(\mathrm{x}^{\prime}\right)\right)}-\frac{1}{\theta(\mathrm{D} 0)}\right] \mathrm{dx}^{\prime}-\mathrm{A} 0
\end{aligned}
$$

where $\theta$ is the conduction velocity restitution function, and (x) serves as a reminder that these APD, DI, and CL are functions of position $\mathrm{x}$ along the cable. D0 was assumed to be large and independent of position $\mathrm{x}$. This implicit equation in $\mathrm{D} 1(\mathrm{x})$ could be differentiated to remove the integral:

$$
\frac{\mathrm{dD} 1(\mathrm{x})}{\mathrm{dx}}=\frac{1}{\theta(\mathrm{D} 1(\mathrm{x}))}-\frac{1}{\theta(\mathrm{D} 0)} .
$$

This equation was solved for D1 numerically using initial value:

$$
\mathrm{D} 1(0)=\mathrm{CL}(0)-\mathrm{A} 0=\mathrm{BCL}-\mathrm{A} 0 .
$$

D2 was solved similarly:

$$
\frac{\mathrm{dD} 2(\mathrm{x})}{\mathrm{dx}}=\frac{1}{\theta(\mathrm{D} 2(\mathrm{x}))}-\frac{1}{\theta(\mathrm{D} 1(\mathrm{x}))}-\frac{\mathrm{dA} 1(\mathrm{x})}{\mathrm{dx}}
$$

using $A 1(x)=f(D 1(x))$, where $f$ was the APD restitution function.

Using these equations, we found that node position 

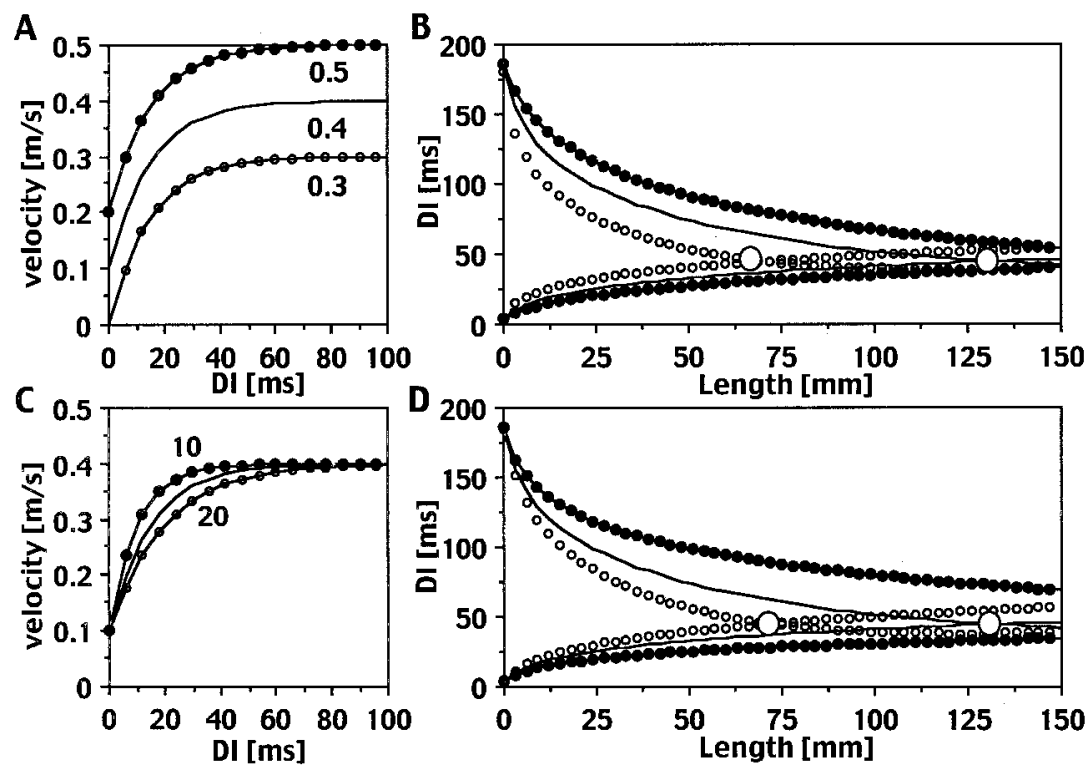

Figure 2. Effect of conduction velocity restitution curve parameters on spatial distribution of diastolic interval (DI) for the first and second beat following basic cycle length shortening from 500 to $300 \mathrm{~ms}$. DI node positions are marked by large unfilled circles. The action potential duration [ms] restitution curve equation was $300-200 \exp (-D I / 50)$. The equation for the conduction velocity [m/s]) restitution curves without circles in panels $A$ and $C$ was $0.4-0.3 \exp (-D I / 15)$. (A,B) Three conduction velocity restitution curves with different maximum values of 0.5 , 0.4 , and 0.3 $[\mathrm{m} / \mathrm{s}](A)$ and their corresponding D1 and D2 spatial distributions $(B)$. When conduction velocity maximum was $0.5,0.4$, and 0.3 , the DI gradients did not intersect within $150 \mathrm{~mm}$, or intersected at 132 and $71 \mathrm{~mm}$, respectively, i.e., lower conduction velocity made nodes occur closer to the stimulus site. (C,D) Range of DI over which velocity varied was also important in determining DI node position. Three conduction velocity restitution curves with different time constants $(10,15,20)(C)$ and their corresponding D1 and D2 spatial distributions $(D)$. When the time constant was 10, 15, or 20, the DI gradients did not intersect within $150 \mathrm{~mm}$, or intersected at 132 and $72 \mathrm{~mm}$, respectively, i.e., conduction velocity change over a wider range of DI made nodes occur closer to the stimulus site.

was affected by two characteristics of the conduction velocity restitution curve, range of DI over which conduction velocity varies, and maximum conduction velocity. Figure 2 shows the effects of each. Panel A shows three conduction velocity restitution curves with fixed time constant and varying maximum conduction velocity $(0.5,0.4,0.3 \mathrm{~m} / \mathrm{sec})$. Panel B shows the D1 and D2 spatial distributions corresponding to the three curves. The middle curve in panel A approximates canine ventricular conduction velocity restitution. When the conduction velocity restitution curve was shifted vertically such that conduction velocity varied over the exact same range of DI, the lower curves produced nodes closer to the stimulus site. This result is expected because lower conduction velocity produces a larger increase in DI as the $\mathrm{S}_{2}$ excitation travels down the cable and concomitantly a larger decrease in DI with $\mathrm{S}_{3}$. The steeper slopes of the spatial distributions then cause a shorter distance to intersection from the stimulus site.

Node position also was affected by the range of DI over which conduction velocity varies. Panel $\mathrm{C}$ shows three conduction velocity restitution curves with fixed maximum and minimum velocity but differing time constants $(10,15$, and $20 \mathrm{msec})$. The middle curve in panel $\mathrm{C}$ approximates canine ventricular conduction velocity restitution. Longer time constants (conduction velocity variation over a longer range of DI) made the nodes occur closer to the stimulus site (panel D). The effect of
DI range on node formation is best understood by considering the limiting case of fixed conduction velocity (infinitely large time constant). In the scenario of singlesite pacing with homogeneous tissue, a constant velocity leads to DI with a value determined solely by stimulus coupling interval and previous APD, which may vary in time depending upon coupling interval but is uniform in space for every beat, thereby precluding node formation. In a similar manner, if conduction velocity varies for only a small range of DI, and such short DIs never occur for particular S1S1, then for that case, conduction velocity would effectively be fixed, and DI spatial distribution would be flat. However, if some of the shorter DIs occur for particular S1S1, then shallow DI spatial distributions could occur and form a node at long distances from the stimulus site.

\section{Evolution of Spatial Distributions with Continued Stimulation}

Earlier, we used mathematical equations describing behavior of independent cable segments to compute DI spatial distributions for $\mathrm{S}_{2}$ and $\mathrm{S}_{3}$. Spatial distributions of DI for further beats could be calculated in like manner, replacing subscripts 1 and 2 in Equation 5 with $\mathrm{n}$ and $\mathrm{n}+1$. However, continued simulation using these equations eventually produces unphysiologically sharp slopes 

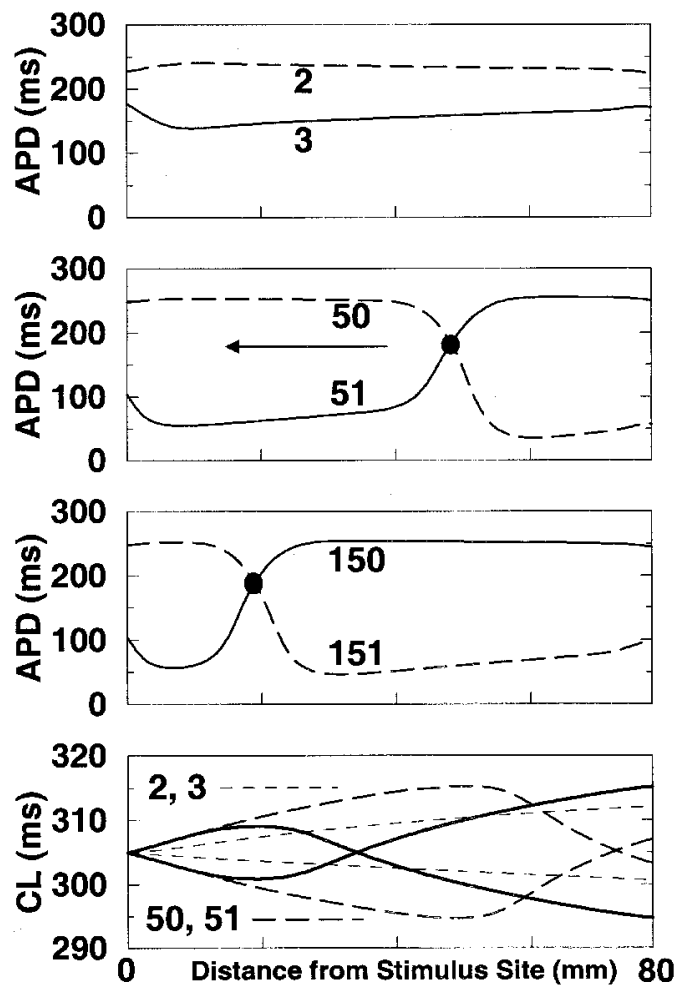

Figure 3. Evolution of action potential duration (APD) spatial distributions for a cable of length $80 \mathrm{~mm}$, paced at a basic cycle length of $310 \mathrm{~ms}$. The cable was paced at the left edge. The spatial distribution for even (hashed) and odd (solid line) beats are shown for stimulus numbers 2, 3 (top panel), 50, 51 (second panel), and at steady state (stimulus numbers 150, 151, third panel). The APD node (spatial distribution intersection) moved leftward with increasing stimulus number. Bottom panel: Evolution of cycle length spatial distributions. The solid lines represent steady-state cycle length $(C L)$ distribution. Similar to APD spatial distribution, the CL node formed at the right end and moved toward the stimulus site.

of DI and APD spatial distributions between the alternans regions of different phase due to lack of inclusion of electrotonic coupling in the equations. We therefore abandon this "discrete" model for the BR model to show how APD spatial distributions evolve with continued pacing. The BR model is more physiologic with respect to electrotonic coupling, but results from it are less transparent mathematically.

Figure 3 shows APD spatial distributions for a cable of length $80 \mathrm{~mm}$ oriented horizontally. The left end of the cable was paced at BCL $=310 \mathrm{msec}$. The top panel shows the APD spatial distributions produced by the 2 nd and 3rd pacing stimuli, the second panel shows the 50th and 51st stimuli, and the third panel shows the steadystate APD spatial distributions (150th and 151st stimuli). The APD nodes are marked with filled circles. The figure shows that initially shallow, nonintersecting spatial distributions can develop into steeper spatial distributions as stimulus number increases. For the simulation shown here, it took $>30$ beats for intersecting APD spatial distributions to form. They formed at the right edge of the cable, gradually moved toward the stimulus site, and eventually stopped at a fixed distance from the stimulus site. The BR model has a conduction velocity variation over only a small range of DI. ${ }^{10}$ This caused the initially shallow APD spatial distributions and slow development of nodes. In addition to DI and APD, CL and conduction velocity spatial distributions also evolved in time. An example is given for CL in the bottom panel, with CL spatial distributions for stimulus numbers $2,3,50,51$, 150 , and 151 (steady state).

\section{Steady-State Spatial Distributions}

The effect of cable length on steady-state APD spatial distributions of the even and odd beats are shown in the top three panels of Figure 4 at BCL $=310 \mathrm{msec}$. As in Figure 3, pacing was applied at the left end. There were 1,2 , and 3 nodes at steady state for cables of length 80 , 117.5 , and $135 \mathrm{~mm}$, respectively. Multiple nodes imply alternating regimes of alternans in or out of phase with the alternans at the stimulus site. It was found that nodes could not exist close to the edge of the tissue at the unstimulated end of the cable, e.g., only one node

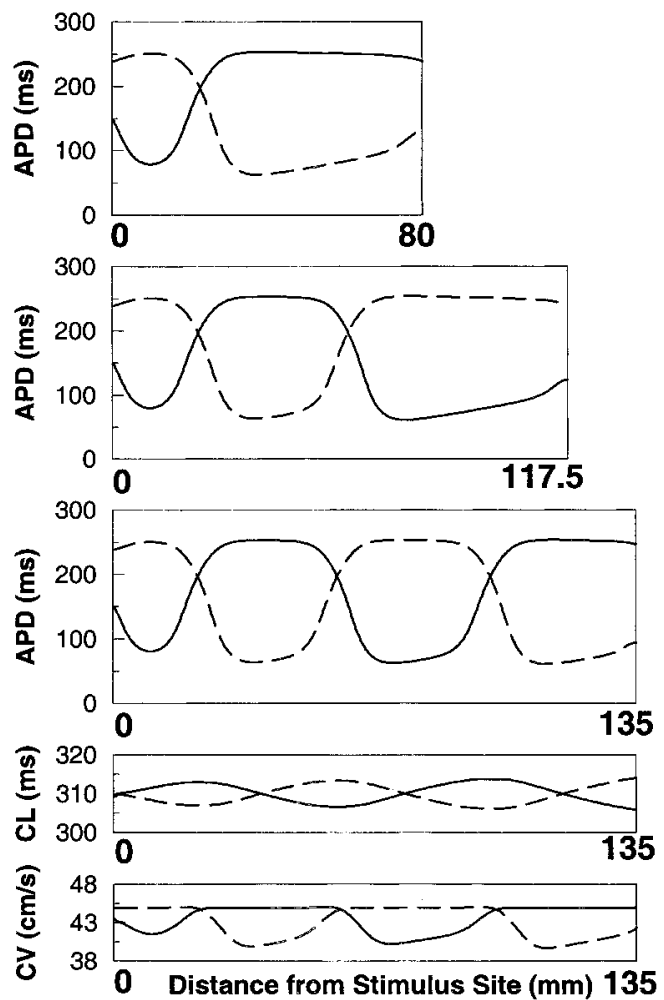

Figure 4. Steady-state action potential duration (APD), cycle length $(C L)$, and conduction velocity $(C V)$ spatial distributions over a cable for even (hashed) and odd (solid line) beats at a basic cycle length of $310 \mathrm{~ms}$. The cable was paced at the left edge. Top three panels: Effect of cable length on APD node position and number. Bottom three panels: Relationship between APD spatial distribution, CL spatial distribution, and CV spatial distributions for a cable length of $135 \mathrm{~mm}$. $C L$ nodes formed at the antinodes of the APD distributions. 

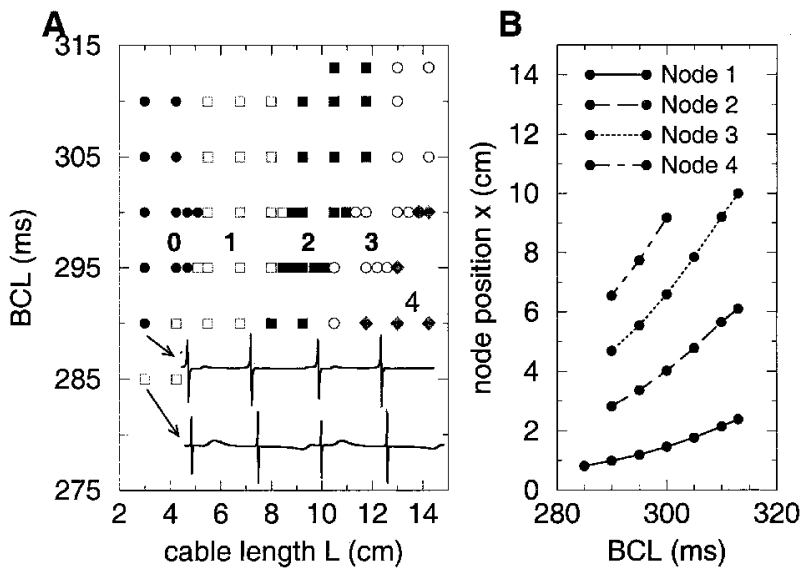

Figure 5. Relationship between action potential duration node position, number of nodes, basic cycle length $(B C L)$, and cable length at steady state. (A) Parameter space is divided into 0 (concordant), 1, 2, 3 , and 4 node regimes. As cycle length is shortened for a given cable length, the number of nodes can increase. ECGs computed for BCL of 290 and $285 \mathrm{~ms}$ at cable length of $3 \mathrm{~cm}$ are also shown. (B) Node position as a function of BCL for cable length of $14 \mathrm{~cm}$. As BCL was reduced, internodal distances decreased.

arose in the $80-\mathrm{mm}$ cable, although judging from the 117.5-mm cable, two nodes might have been expected to fit in the $80-\mathrm{mm}$ cable.

The three lower panels of Figure 4 show the relationship between spatial distributions of APD, CL, and conduction velocity at steady state for even and odd beats for a cable length of $135 \mathrm{~mm}$ and $\mathrm{BCL}=310 \mathrm{msec}$. Under these conditions, maximum CL and conduction velocity alternation were approximately $6 \mathrm{msec}$ and $50 \mathrm{~mm} / \mathrm{sec}$, respectively. CL nodes occurred at the antinodes of DI, APD, and conduction velocity spatial distributions.

\section{Relationship Between BCL and Node Position and Number}

The dependence of number of nodes on cable length and BCL was studied by running simulations with various combinations of cable length and BCL (Fig. 5A). In general, longer cable lengths and shorter BCL allowed greater numbers of nodes. For example, pacing a cable of 3 -cm length at $\mathrm{BCL}=310-290 \mathrm{msec}$ produces concordant alternans (no nodes). Shortening BCL to 285 msec causes a node (discordant alternans) to appear. This example mimics the experimental finding of Pastore et al., 8 who demonstrated that shortening of BCL could cause concordant alternans to become discordant. Representative ECGs computed from two electrodes placed one above the other near the center of a $3-\mathrm{cm}$ cable for $\mathrm{BCL}=290$ and $285 \mathrm{msec}$ also are shown at the bottom of the figure. Both ECGs exhibit QRS and TWA. The lower ECG exhibits $\mathrm{T}$ waves that alternate in polarity.

Figure 5B shows steady-state APD node position versus BCL for a fixed cable length of $140 \mathrm{~mm}$. Steady-state node positions move closer to the paced end of the cable and internodal distances shrink as BCL is reduced. The nodes are at equal distances from each other for a given BCL.

\section{Restitution Curve During Discordant Alternans}

In theory, it is not possible for APD to be identical on more than three consecutive beats, such as at the steadystate APD node, if CL alternates at that position (fourth panel, Fig. 4), and if DI uniquely determines APD as in standard (S1S2) restitution curves. That is because if DI uniquely determines APD, the sequence of DI and APD at the steady-state APD node would be a repetition of $\mathrm{DI}^{*} \rightarrow \mathrm{APD}^{*} \rightarrow \mathrm{DI}^{*} \rightarrow$ APD*, where the asterisk indicates the putative steady-state value at the node. CL then is a fixed value APD* + DI* and obviously does not alternate. Graphing the DI, APD relationship curves that arise during discordant alternans, i.e., dynamic restitution curves (Fig. 6) shows that the paradox lies in assumption of a fixed restitution curve. The standard restitution curve (dotted line) and two pairs of dynamic restitution curves are shown, from the 50th and 51st beats (thin solid and hashed lines), and one pair at steady state (thick solid and hashed lines). The dynamic restitution curves are significantly lower than the standard restitution curve for mid-range DI. The bottom inset is an enlarged view of the two sets of dynamic restitution curves where they intersect the two sets of lines representing the equations $\mathrm{DI}+\mathrm{APD}=\mathrm{CL}$, again, one set for the CLs produced by $\mathrm{S}_{50} \sim \mathrm{S}_{51}$ and $\mathrm{S}_{51} \sim \mathrm{S}_{52}$, and the other set at steady state. The split between the dynamic restitution curves expands with time (thick lines are wider apart than thin lines), whereas the split between the dynamic CL lines contract (thick lines are closer together than thin lines). The unfilled circles indicate the steady-state APD node value and corresponding DIs.

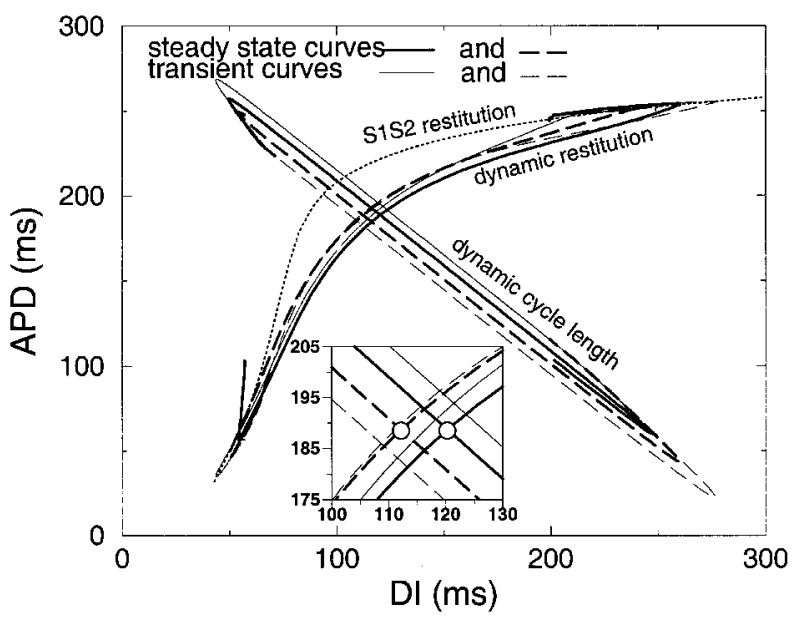

Figure 6. Two sets of dynamic restitution curves and two sets of dynamic cycle length lines during discordant alternans in a cable 62.5 $\mathrm{mm}$ long paced at a basic cycle length of $305 \mathrm{~ms}$. Thin lines $=$ stimulus numbers 50 and 51; thick lines $=$ steady state; hashed lines $=$ even numbered stimuli; solid lines $=$ odd numbered stimuli. Inset: Enlarged view of intersection of restitution and cycle length lines. 

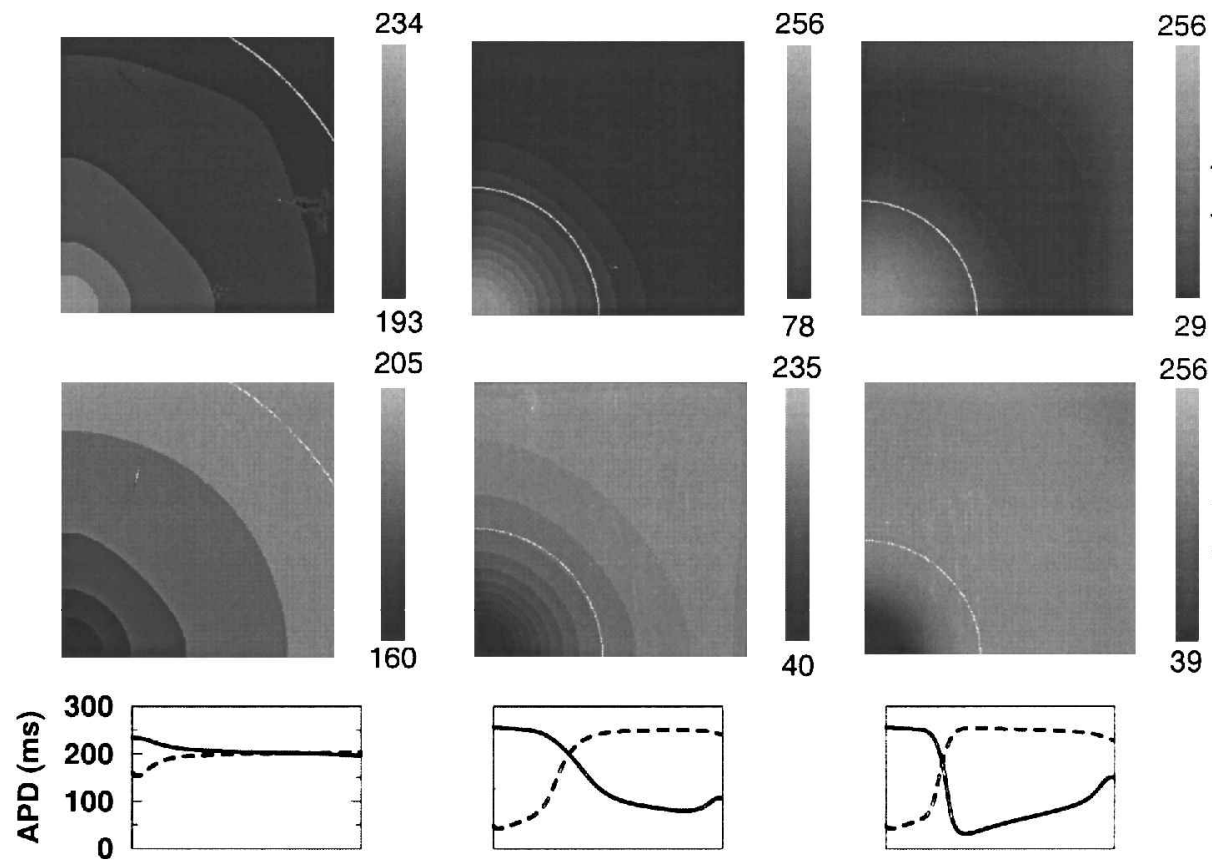

Figure 7. Action potential duration (APD) spatial distributions in an $80 \times 80 \mathrm{~mm}$ two-dimensional sheet produced by a point stimulus applied at the bottom left corner every $310 \mathrm{~ms}$. From left to right, top row: APD spatial distribution for stimulus numbers 2, 20, and 52; center row: APD spatial distribution for stimulus numbers 3,21, and 53; bottom row: APD spatial distributions on the diagonal of the two-dimensional sheets. The white lines in the two-dimensional sheets indicate APD node line position where APD was fixed for consecutive beats. When stimulation was continued, the APD node line became a quarter circle (a line equidistant from stimulus site).

They show how DI can alternate yet still produce the same APD. The true steady-state sequence at the APD node (value $\mathrm{APD}^{*}$ ) for one full cycle is short $\mathrm{DI} \rightarrow$ $\mathrm{APD}^{*}$ - long $\mathrm{CL} \rightarrow$ long $\mathrm{DI} \rightarrow \mathrm{APD}^{*}-$ short $\mathrm{CL} \rightarrow$ short DI, and the paradox is resolved.

The APD node position can be fixed in space, only if the difference in alternating cycle lengths $(\Delta \mathrm{CL})$ can match the difference in DI $(\Delta \mathrm{DI})$ of the dynamic restitution curves at that position. Initially, the APD node position is unstable and moves toward the stimulus site because $\Delta \mathrm{CL}>\Delta \mathrm{DI}$. $\Delta \mathrm{DI}$ is determined by electrotonic coupling and the standard APD restitution curve and gradually increases. $\Delta \mathrm{CL}$ initially is large and gradually decreases. The APD node stops moving toward the stimulus end of the cable when $\Delta \mathrm{CL}=\Delta \mathrm{DI}$. In mathematical notation, this relationship is expressed by Equation 6:

$$
\Delta \mathrm{DI}=\int_{0}^{\mathrm{x}}\left[\frac{1}{\theta\left(\mathrm{D}[\mathrm{n}+1]\left(\mathrm{x}^{\prime}\right)\right)}-\frac{1}{\theta\left(\mathrm{D}[\mathrm{n}]\left(\mathrm{x}^{\prime}\right)\right)}\right] \mathrm{dx^{ \prime }} .
$$

We can make a rough approximation of APD node position $\mathrm{x}$ by Equation 7:

$$
\mathrm{x} \cong \Delta \mathrm{DI}\left(\frac{\theta \max \cdot \theta \min }{\theta \max -\theta \min }\right)
$$

where $\theta \max$ is the asymptote of the conduction velocity restitution curve and $\theta \mathrm{min}$ is the minimum value. Equation 7 predicts among other things that if conduction velocity is independent of DI and is constant, the node is at an infinitely long distance from the stimulus site and cannot exist. For a fixed difference between maximum and minimum conduction velocity, the smaller conduction velocity produces a shorter distance to the node.

The slope of the dynamic restitution curves are nearly 1 at the intersections with the two diagonal lines representing DI $+\mathrm{APD}=$ long and $\mathrm{DI}+\mathrm{APD}=$ short $\mathrm{CL}$ (Fig. 6). It can be shown mathematically that if the product of the slopes of the linearized dynamic restitution curves are 1 (including the case where they are both 1), then APD values that flank APD* can alternate stably. It is important to note that the different dynamic restitution curves do not signify an a priori spatially heterogenous distribution of restitution curves, but a dynamic modulation of inherent restitution properties due to the electrotonic coupling present both in cardiac tissue and in our computer models with Laplacian coupling.

\section{Two-Dimensional Simulation and Results from Other Models}

Figure 7 shows how discordant alternans forms in a two-dimensional sheet paced at the bottom left-hand corner. APD spatial distributions over the sheet are shown for even (top panels) and odd (lower panels) numbered stimuli in the beginning (left panels), at an intermediate length of time (middle panels), and near 
steady state (right panels). The APD node in the onedimensional cable was a point, a fixed distance from the stimulus site. In two dimensions, the APD node formed a line. It moved toward and stabilized near the pacing site. The APD spatial distribution on the diagonal line of the two-dimensional sheet is shown at the very bottom.

Simulations of discordant alternans in other models ${ }^{10-12}$ produced results qualitatively similar to simulations from the BR model, demonstrating the robustness of mechanisms of initiation and evolution across different models, as might be expected from the simple mechanism that we proposed. One quantitative difference was of note. In the BR model, QRS alternans and TWA arose simultaneously, due to similar DI over which APD and conduction velocity restitution had large slopes, whereas in the other models, TWA could precede QRS alternans with rate increase, depending on the relative position of the bend in the two curves.

We introduced a smooth gradient (Fig. 8, panels B and C) or a small segment of altered calcium current kinetics (panel D) into the cable properties to see how small inhomogeneities of ionic properties would affect discordant alternans. Panel A shows the steady-state APD alternans in control. When the calcium current kinetics was graded over the cable, APD node and discordant alternans formation was suppressed and alternans was concordant. The gradient was introduced by multiplying the voltage-dependent time constants for calcium current activation and inactivation in the BR model, $\tau_{\mathrm{d}}(\mathrm{V})$ and $\tau_{\mathrm{f}}(\mathrm{V})$, respectively, by a function $\mathrm{b}(\mathrm{x})=0.9+$ $0.1 \mathrm{x} / \mathrm{L}$, which varies linearly from 0.9 at the pacing end of the cable $(x=0)$, corresponding to faster calcium kinetics, to 1.0 at the other end $(\mathrm{x}=\mathrm{L})$. When this gradient was reversed so the time constants were normal at the stimulus end and linearly decreased to $85 \%$ of their value at the nonstimulated end of the cable, i.e., $\mathrm{b}(\mathrm{x})=$ $1.0-0.15^{*} \mathrm{x} / \mathrm{L}$, the position of the node was shifted away from the stimulus site compared to control, and alternans ceased at the nonstimulated end. When the calcium kinetics was slowed down inside the segment represented by the bar in panel $\mathrm{D}$, i.e., $\mathrm{b}(\mathrm{x})=1.15$ for $4.25 \mathrm{~cm}<\mathrm{x}<5.5 \mathrm{~cm}$ and $\mathrm{b}(\mathrm{x})=1.0$ elsewhere, the position of the node migrated to that segment. Tissue heterogeneities thus were capable of suppressing, facilitating, or pinning location of the nodes.

\section{Discussion}

There have been many experimental studies of the mechanism of cardiac alternans, whether of APD or mechanical contraction (reviewed by Surawicz and Fisch ${ }^{13}$ ), and equally many studies into the correlation between alternans and ischemia (reviewed by Verrier and Nearing ${ }^{2}$ ), or long QT syndrome. ${ }^{3,14,15}$ Theoretical work suggests that concordant APD alternans is sustained when the maximum slope of APD restitution is $>1$ and $\mathrm{BCL}$ is short, ${ }^{16-18}$ although there are experiments showing some discrepancy from theory, ${ }^{19}$ and some experimental ${ }^{20,21}$ and theoretical ${ }^{22}$ studies ascribe a more important role to the slope of dynamic rather than stan-
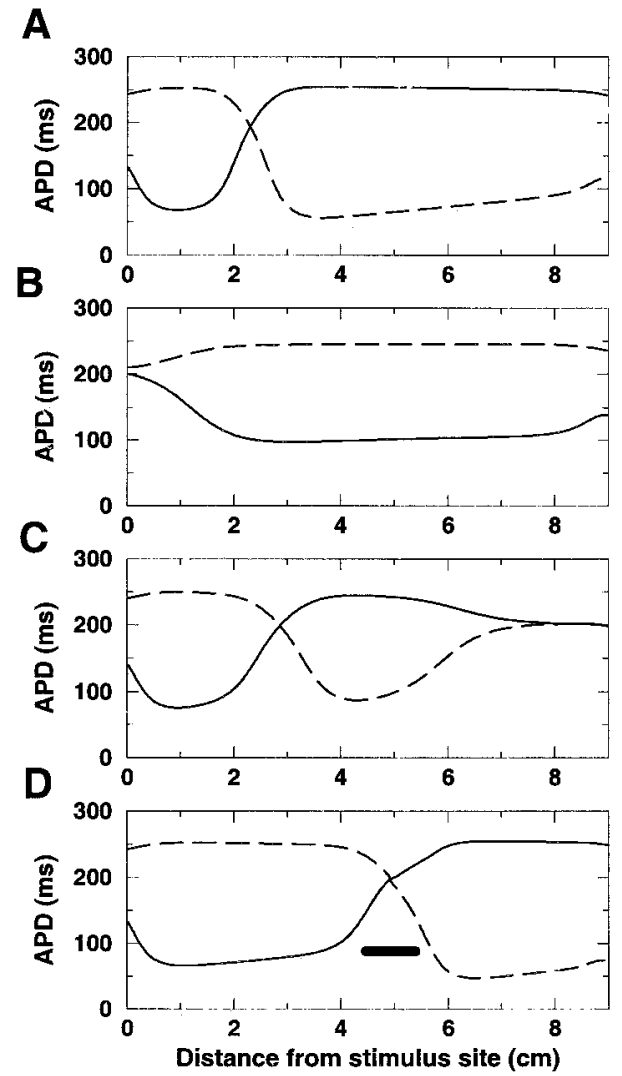

Figure 8. Effects of spatial inhomogeneity on node position. (A) Steady-state action potential duration (APD) spatial distribution for even (hashed) and odd (solid line) beats in the uniform ion channel configuration. $(B, C)$ Steady-state APD spatial distribution in presence of a spatial gradient of calcium kinetics (see text). In panel B, the calcium kinetics is faster than normal at the stimulus end and normal at the nonstimulated end, and alternans remain concordant. With a reverse gradient (panel C), the position of the node was shifted away from the stimulus site compared to control. (D) Steady-state APD spatial distribution in the presence of a small segment (represented by black bar) with slower calcium kinetics (see text). The position of the node migrated to that segment. Tissue heterogeneities thus are capable of suppressing, facilitating, or pinning node positions.

dard restitution curve in alternans production. The APD restitution curve of the BR model as measured with an S1S2 protocol has a maximum slope that is $>1$, and stable alternans was produced in our simulations at the pacing site as expected. In mathematical models, steep restitution curve slope also has been shown to produce complex oscillations in the period of reentry ${ }^{23}$ and spiral wave breakup, ${ }^{10,24-26}$ a phenomenon believed to be an analogue for ventricular fibrillation. The slope of the restitution curve is thus an indirect link between ventricular fibrillation and alternans and may be related to the close relationship between TWA and ventricular fibrillation.

In contrast to concordant alternans, discordant alternans is a less studied phenomenon ${ }^{13,27}$ in cardiac tissue where its mechanism is unknown. In the present study, we focused on the conditions necessary to produce dis- 
cordant alternans in spatially uniform tissue. In such uniform tissue, discordant alternans had to come from a nonuniform initial distribution of DI in space coupled with APD restitution. There were two ways that this could be achieved. The first method required depolarizations to occur at two sites (ectopic focus scenario), but did not require conduction velocity restitution. The spatial distribution of DI produced in the wake of the sinus and ectopic depolarization was sufficient to induce discordant alternans. The second method involved relatively large tissue size, slow conduction velocity, dependence of conduction velocity on DI, and a rate increase. The boundary between the alternans of different phases was represented by nodes where APD was identical on two consecutive beats. APD nodes formed, moved toward the stimulus site, and asymptotically stopped at a distance determined by BCL and cable length. Unlike the ectopic focus scenario, multiple nodes were possible in the sinus node scenario. Multiple nodes indicated multiple regimes in space where the phase of APD alternans was alternately in or out of phase with the alternans phase at the stimulus site. The number of nodes seen depended partially on tissue length. Although only single nodes have been observed experimentally, ${ }^{8}$ this study predicts that more may be found if larger tissues are studied. Simulations also successfully replicated the experimental observation of faster pacing converting concordant to discordant alternans.

During discordant alternans produced by the second scenario, there was alternans not only of DI and APD, but also of CL and conduction velocity. The relative importance of APD alternans and conduction velocity alternans in producing ECG alternans has been debated, with some giving alternans of conduction the primary role $^{7}$ and others, alternans of APD. ${ }^{28-30}$ Two recent studies support primacy of APD alternans; activation times were identical from beat to beat. ${ }^{31,32}$ Appearance of repolarization alternans at slower heart rate than depolarization alternans ${ }^{8}$ also might be interpreted by some that conduction velocity is of lesser importance, although one would naturally expect repolarization (APD) alternans to occur at slower heart rates than depolarization (conduction velocity) alternans in normal cardiac tissue, because of the shorter DI at which conduction velocity varies. However, at the high pacing rates at which discordant alternans is seen, DI typically is small, and effects of conduction velocity cannot be separated from effects of repolarization. In contrast, in a recent study 33 of the induction of ventricular fibrillation by rapid pacing in the dog and Luo-Rudy I ionic model, it was concluded that conduction velocity was important for producing spatial inhomogeneities of CL and DI. The results of our simulations show that both the sinus node and ectopic focus scenarios are valid ways of initiating discordant alternans in homogeneous tissue, and that conduction velocity restitution is crucial in the former, but not necessary in the latter.

We attributed the split between the two dynamic restitution curves to electrotonic coupling, because all ionic properties such as voltage and time dependence of
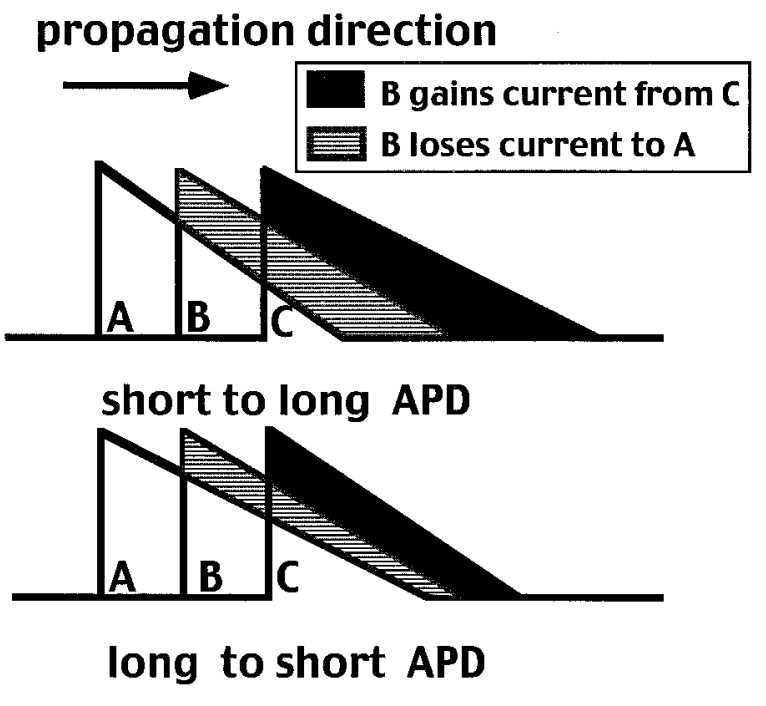

Figure 9. Difference in electrotonic current as a mechanism for splitting of dynamic restitution. Top: Three cable segments of increasing action potential duration (APD) arrayed from left to right. Bottom: The three cable segments in the top panel rearranged so APD decreases from left to right. As excitation propagates from left to right, the difference in membrane voltage between the segments causes segment $B$ to receive electrotonic current from segment $C$ and to lose electrotonic current to A. A comparison of the top and bottom diagrams show that the difference in membrane voltages between the segments (shaded areas) have different morphologies. The net electrotonic current received by segment $B$ therefore is different depending on depolarization direction, increasing or decreasing APD of segment B from its value in the uncoupled state.

ionic channel properties were set uniformly across the tissue in our simulations and because of known lack of cardiac memory in the ionic models we studied. We hypothesized that there was an asymmetry of electrotonic coupling with respect to propagation direction for a given APD spatial distribution (Fig. 9). Consider three adjacent segments in a cable, A, B, and C, with DIdependent APD values $\mathrm{APD}_{\text {shor, }}$ APD $\mathrm{P}_{\text {medium, }}$, and $\mathrm{APD}$ long, in the uncoupled state. If the excitation sequence is $\mathrm{A} \rightarrow \mathrm{B} \rightarrow \mathrm{C}$, segment $\mathrm{B}$ gives current to A but receives current from $\mathrm{C}$ because electrotonic current flows from higher voltage to lower voltage. If propagation direction and velocity are the same, but APD is reversed for segments $\mathrm{A}$ and $\mathrm{C}$ so that $\mathrm{A}, \mathrm{B}$, and $\mathrm{C}$ have DI-dependent $\mathrm{APD}$ values $\mathrm{APD}_{\text {long }}, \mathrm{APD}_{\text {medium }}$, and $\mathrm{APD}_{\text {short }}$, segment $\mathrm{B}$ still gives current to $\mathrm{A}$ and receives current from $\mathrm{C}$, but the duration and magnitude of these currents is different from that of the previous situation, as can be seen in the different shapes and sizes of the overlapping areas. The net electrotonic current received by segment $\mathrm{B}$ therefore is different depending on depolarization direction, even if the uncoupled scalar, DI-dependent values of the three segments remain the same. If more current is received than is lost by segment B, the resulting APD of segment will be longer; if more current is lost than received, the resulting APD will be shorter compared with APD of segment B in the uncoupled state. The split of restitution 
curves was most marked where APD spatial distribution was steep, near the node, as might be expected by this reasoning.

There are other hypotheses concerning the mechanism of discordant alternans. Based on their experimental finding that alternans was frequently of opposite phase at the base and apex of heart regardless of pacing site, coupled with the fact that repolarization properties are known to vary from base to apex, Pastore et al. ${ }^{8}$ made the case that regional differences in repolarization current were important for discordant alternans development. We found that tissue heterogeneity was not necessary for discordant alternans initiation, although it could affect node position. A small change in calcium current characteristics was able to move the node closer to or farther from the pacing site, or facilitate or suppress node formation. Our observation that a small spatial heterogeneity could pin the node position may explain the tendency for discordant alternans to align in a particular direction.

Our study had several limitations. We presented mainly behavior of spatially homogeneous tissue because its behavior was already highly complex, although cardiac tissue is well known to be heterogeneous. It is our hope that our study can serve as a control for future studies of effects of heterogeneity. The ectopic focus scenario also warranted more study than was presented here. Another limitation was our use of only computationally tractable models. A problem of the BR model is that the range of DI over which APD and conduction velocity change is similar; therefore, we did not see repolarization alternans separately from depolarization alternans in the ECG or waveforms. However, this separation was seen in the Noble and mesoscopic models. Finally, cardiac memory was not present in the models we used for our simulations.

With respect to clinical implications, previous theory predicts that concordant alternans could be prevented by reducing heart rate or by using pharmacologic agents that flatten APD restitution curve slope. ${ }^{20,25,26,32}$ Results from the present study suggest that discordant alternans that arises by the second scenario could be prevented by drugs that increase conduction velocity or reduce range of DI over which conduction velocity varies, even if concordant alternans is not preventable.

\section{Conclusion}

Discordant alternans can form spontaneously in spatially homogeneous tissue paced at high frequency due to the interaction of conduction velocity and APD restitution, or due to the repolarization gradient produced by an ectopic beat. The dynamic splitting of APD restitution curves due to electrotonic coupling plays a crucial role in the stability of the boundaries that separates regions of alternans with opposite phase.

\section{References}

1. Kimura E, Yoshida K: A case showing electrical alternans of the T wave without change in the QRS complex. Am Heart J 1963;65: 391-395.
2. Verrier RL, Nearing BD: Electrophysiologic basis for $T$ wave alternans as an index of vulnerability to ventricular fibrillation. J Cardiovasc Electrophysiol 1994;5:445-461.

3. Schwartz PJ, Malliani A: Electrical alternans of the T-wave: Clinical and experimental evidence of its relationship with the sympathetic nervous system and with the long Q-T syndrome. Am Heart J 1975;89:45-50.

4. Smith JM, Clancy EA, Valeri R, Ruskin JN, Cohen RJ: Electrical alternans and cardiac electrical instability. Circulation 1988;77: 110-121.

5. Rosenbaum DS, Jackson LE, Smith JM, Garan H, Ruskin JN, Cohen RJ: Electrical alternans and vulnerability to ventricular arrhythmias. N Engl J Med 1994;330:235-241.

6. Estes NAM, Michaud G, Zipes DP, El-Sherif N, Venditti FJ, Rosenbaum DS, Albrecht P, Wang PJ, Cohen RJ: Electrical alternans during rest and exercise as predictors of vulnerability to ventricular arrhythmias. Am J Cardiol 1997;80:1314-1318.

7. Downar E, Manse M, Durrer D: The effect of acute coronary artery occlusion on subepicardial transmembrane potentials in the intact heart. Circulation 1977;56:217-224.

8. Pastore JM, Girouard SD, Laurita KR, Akar FG, Rosenbaum DS: Mechanism linking T-wave alternans to the genesis of cardiac fibrillation. Circulation 1999;99:1385-1394.

9. Beeler GW, Reuter HO: Reconstruction of the action potential of ventricular myocardial fibres. J Physiol 1977;268:177-121.

10. Fenton F, Karma A: Vortex dynamics in three-dimensional continuous myocardium with fiber rotation: Filament instability and fibrillation. Chaos 1998;8:20-47.

11. Noble D: A modification of the Hodgkin-Huxley equations applicable to Purkinje fibre action and pace-maker poentials. J Physiol 1962;160:317-352.

12. Karma A: Electrical alternans and spiral wave breakup in cardiac tissue. Chaos 1994;4:461-472.

13. Surawicz B, Fisch C: Cardiac alternans: Diverse mechanisms and clinical manifestations. J Am Coll Cardiol 1992;20:483-499.

14. Hiejima K, Sano T: Electrical alternans of the TU wave in Romano-Ward syndrome. Br Heart J 1976;38:767.

15. Rosenbaum MB, Acunzo RS: Pseudo 2:1 atrioventricular block and $\mathrm{T}$ wave alternans in the long QT syndromes. J Am Coll Cardiol 1991;18:1363-1366.

16. Nolasco JB, Dahlen RW: A graphic method for the study of alternation in cardiac action potentials. J Appl Physiol 1968;25: 191-196.

17. Watanabe M, Otani NF, Gilmour RF Jr: Biphasic restitution of action potential duration and complex dynamics in ventricular myocardium. Circ Res 1995;76:915-921.

18. Guevara MR, Glass L: Electrical alternans and period doubling bifurcations. Comput Cardiol 1984;167-170.

19. Hall GM, Bahar S, Gauthier DJ: Prevalence of rate-dependent behaviors in cardiac muscle. Phys Rev Lett 1999;82:2995-2998.

20. Koller ML, Riccio ML, Gilmour RF Jr: Dynamic restituion of action potential duration during electrical alternans and ventricular fibrillation. Am J Physiol 1998;275:H1635-H1642.

21. Riccio ML, Koller ML, Gilmour RF Jr: Electrical restitution and spatiotemporal organization during ventricular fibrillation. Circ Res 1999;84:955-963.

22. Fenton FH, Evans SJ, Hastings HM: Memory in an excitable medium: A mechanism for spiral wave breakup in the low-excitability limit. Phys Rev Lett 1999;83:3964-3967.

23. Courtemanche M, Glass L, Keener JP: Instabilities of a propagating pulse in a ring of excitable media. Phys Rev Lett 1993;70: 2182-2185.

24. Qu Z, Weiss JN, Garfinkel A: Cardiac electrical restitution properties and stability of reentrant spiral waves: A simulation study. Am J Physiol 1999;276:H269-H283.

25. Garfinkel A, Kim Y-H, Voroshilovsky O, Qu Z, Kil JR, Lee M-H, Karaquezian HS, Weiss JN, Chen P-S: Preventing ventricular fibrillation by flattening cardiac restitution. Proc Natl Acad Sci U S A 2000;97:6061-6066.

26. Karma A: New paradigm for drug therapies of cardiac fibrillation. Proc Natl Acad Sci U S A 2000;97:5687-5689.

27. Hellerstein HK, Liebow JM: Electrical alternation in experimental coronary artery occlusion. Am J Physiol 1950;160:366-374. 
28. Hoffman BF, Suckling EE: Effect of heart rate on cardiac membrane potentials and unipolar electrogram Am J Physiol 1954;179: 123-130.

29. Kleinfeld M, Stein E: Electrical alternans of components of the action potential. Am Heart J 1968;75:528-530.

30. Dilly SG, Lab MJ: Electrophysiological alternans and restitution during acute regional ischemia in myocardium of anesthetized pig. J Physiol (Lond) 1988;402:315-333.

31. Shimizu W, Antzelevitch C: Cellular and ionic basis for T-wave alternans under long-QT conditions. Circulation 1999;99:14991507.

32. Hirayama Y, Saitoh H, Atarashi H, Hayakawa H: Electrical and mechanical alternans in canine myocardium in vivo. Circulation 1993;88:2894-2902.

33. Cao J-M, Qu Z, Kim Y-H, Wu W-J, Garfinkel A, Weiss JN, Karagueuzian HS, Chen P-S: Spatiotemporal heterogeneity in the induction of ventricular fibrillation by rapid pacing: Importance of cardiac restitution properties. Circ Res 1999;84:1318-1331.

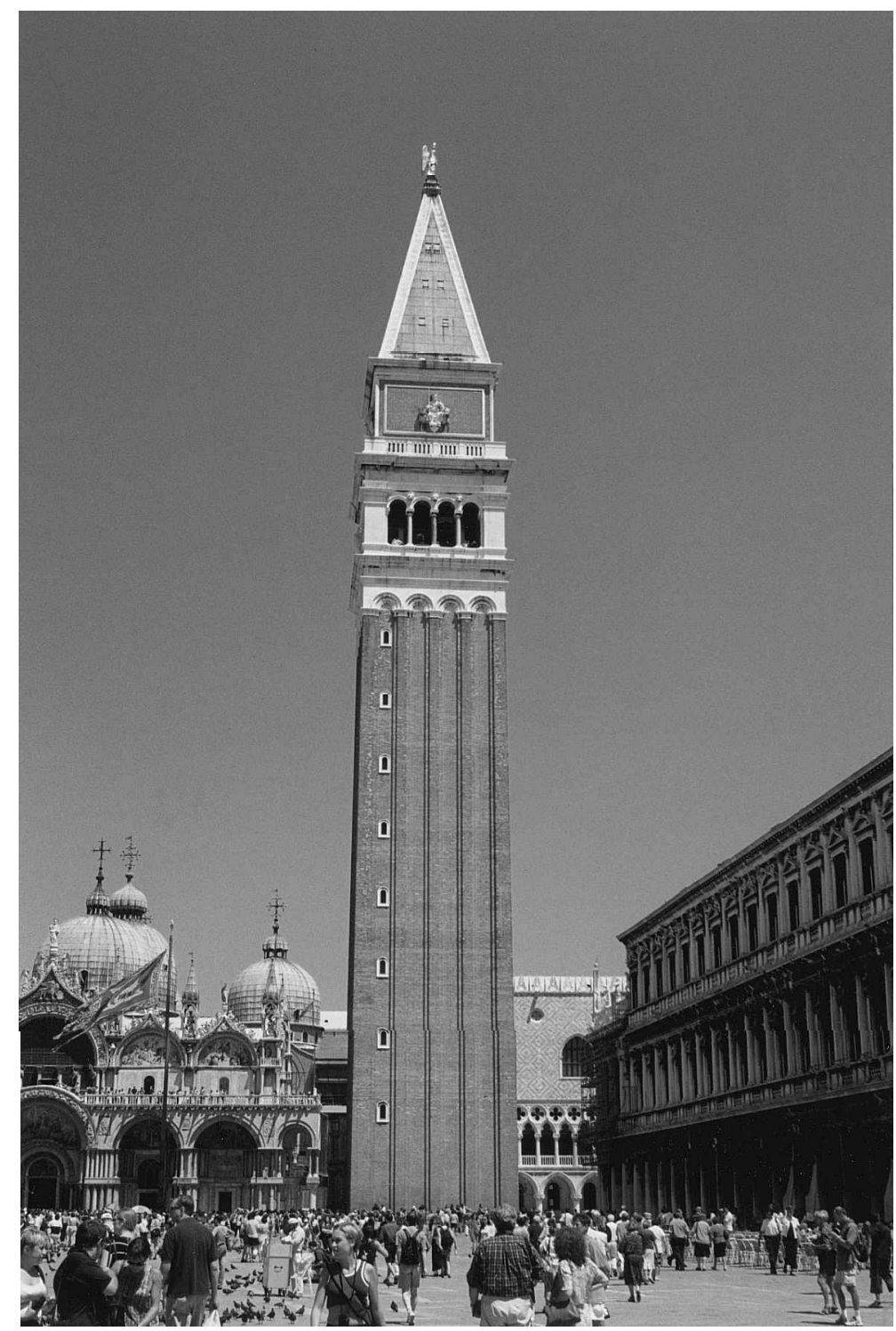

Campanile Piazza, San Marco, Venice 